\title{
Stop or go? Preventive cognitive therapy with guided tapering of antidepressants during pregnancy: study protocol of a pragmatic multicentre non-inferiority randomized controlled trial
}

Nina M. Molenaar ${ }^{2 \dagger}$, Marlies E. Brouwer ${ }^{1 \dagger}$, Claudi L. H. Bockting ${ }^{1}$, Gouke J. Bonsel ${ }^{3}$, Christine N. van der Veere ${ }^{4}$, Hanneke W. Torij ${ }^{5}$, Witte J. G. Hoogendijk2 ${ }^{2}$, Johannes J. Duvekot ${ }^{3}$, Huibert Burger ${ }^{6}$ and

Mijke P. Lambregtse-van den Berg $2,3,7^{*}$

\begin{abstract}
Background: Approximately $6.2 \%$ of women in the USA and $3.7 \%$ of women in the UK, use Selective Serotonin Reuptake Inhibitors (SSRIs) during their pregnancies because of depression and/or anxiety. In the Netherlands, this prevalence is around $2 \%$. Nonetheless, SSRI use during pregnancy is still controversial. On the one hand SSRIs may be toxic to the intrauterine developing child, while on the other hand relapse or recurrence of depression during pregnancy poses risks for both mother and child. Among patients and professionals there is an urgent need for evidence from randomized studies to make rational decisions regarding continuation or tapering of SSRIs during pregnancy. At present, no such studies exist.

Methods/Design: 'Stop or Go' is a pragmatic multicentre randomized non-inferiority trial among 200 pregnant women with a gestational age of less than 16 weeks who use SSRIs without clinically relevant depressive symptoms. Women allocated to the intervention group will receive preventive cognitive therapy with gradual, guided discontinuation of SSRIs under medical management (STOP). Women in the control group will continue the use of SSRIs (GO). Primary outcome will be the (cumulative) incidence of relapse or recurrence of maternal depressive disorder (as assessed by the Structured Clinical Interview for DSM disorders) during pregnancy and up to three months postpartum. Secondary outcomes will be child outcome (neonatal outcomes and psychomotor and behavioural outcomes up to 24 months postpartum), and health-care costs. Total study duration for participants will be therefore be 30 months. We specified a non-inferiority margin of $15 \%$ difference in relapse risk.

(Continued on next page)
\end{abstract}

\footnotetext{
* Correspondence: mijke.vandenberg@erasmusmc.nl

${ }^{\dagger}$ Equal contributors

2Department of Psychiatry, Erasmus Medical Centre, 's Gravendijkwal 230,

3015 CE Rotterdam, The Netherlands

${ }^{3}$ Department of Obstetrics and Gynaecology, Erasmus Medical Centre, 's

Gravendijkwal 230, 3015 CE Rotterdam, The Netherlands

Full list of author information is available at the end of the article
} 
(Continued from previous page)

Discussion: This study is the first to investigate the effect of guided tapering of SSRIs with preventive cognitive therapy from early pregnancy onwards as compared to continuation of SSRIs during pregnancy. We will study the effects on both mother and child with a pragmatic approach. Additionally, the study examines cost effectiveness. If non-inferiority of preventive cognitive therapy with guided tapering of SSRIs compared to intended continuation of SSRIs is demonstrated for the primary outcome, this may be the preferential strategy during pregnancy.

Trial registration: Netherlands Trial Register (NTR): NTR4694; registration date: 16-jul-2014

Keywords: Pregnancy, Antidepressants, Selective serotonin reuptake inhibitors, SSRI, Depression, Prevention, Relapse, Recurrence, Cognitive therapy, Perinatal outcome, Cost-effectiveness

\section{Background}

Depressive disorder and anxiety disorders are the primary indications for the use of Selective Serotonin Reuptake Inhibitors (SSRIs). Worldwide, the SSRI prescription rate during pregnancy ranges from $6.2 \%$ in the USA [1], to $3.7 \%$ in the UK [2]. The actual Dutch nationwide estimated use of SSRIs during pregnancy is about two percent $[3,4]$; while in the Rotterdam area this number is even as high as five percent [5]. Nonetheless, SSRI use during pregnancy is still controversial. On the one hand SSRIs may be toxic to the intrauterine developing child, while on the other hand, relapse of depression and/or anxiety during pregnancy poses risks for both mother and child [6].

The preventive effect of SSRIs for relapse of depression during pregnancy seems equivocal. One naturalistic study showed a significant increased risk of relapse in pregnant women who discontinued their medication compared to continuing medication (68\% vs. $26 \%$ ), while another naturalistic study showed no clear difference relapse rates of depression (16\% in total) between pregnant women continuing or discontinuing antidepressants $[7,8]$.

Pregnancy-related complications both exist for women using SSRIs during pregnancy and women with untreated depression/anxiety during pregnancy, posing a dilemma for the treating physician who considers SSRI withdrawal. For example, studies found significantly increased risks for preeclampsia among women who use SSRIs and increased risks for pregnancy-induced hypertension in women with depression/anxiety during pregnancy compared to healthy controls $[9,10]$.

Whether or not SSRIs are of direct influence on the newborn, both short- and long-term, is another unresolved issue. For example, a recent meta-analysis showed an increased risk for cardiovascular malformations $(\mathrm{RR}=$ 1.36) and septal heart defects $(R R=1.40)$ with use of SSRIs [11]. These findings were however not supported by a recent Nordic cohort study, which - after a sibling controlled analysis - found no substantial increase in prevalence of overall cardiac birth defects for any SSRI
$(\mathrm{OR}=0.92)$ [12]. Another example of evidence of a potential direct toxic effect is the association of SSRI use with persistent pulmonary hypertension (PPHN) of the neonate. A large cohort study from the Scandinavian national health registers showed a twofold-increased risk of PPHN with exposure later than gestational week $20(\mathrm{OR}=2.1)$ [13]. However, this risk appeared more modest $(\mathrm{OR}=$ 1.51) in a large cohort study from 46 US states [14].

Several other effects of SSRIs during pregnancy have been described, such as a higher risk of poor neonatal adaptation $(\mathrm{OR}=5.07)$, respiratory distress $(\mathrm{OR}=2.20)$, tremors $(\mathrm{OR}=7.89)$, preterm delivery and small for gestational age, lower birth weight and lower Apgar scores at 1 and 5 min after birth $[15,16]$. Long-term effects on children are less often investigated. One systematic review found an adverse effect on children's motor development but not on emotional or behavioural development [17]. Two large studies reported on the association between maternal SSRI use and childhood autism spectrum disorders, but found conflicting results $[18,19]$.

On the other hand, leaving depression or anxiety disorders untreated may be hazardous to the unborn child as well. At present, it is well known that children of women who suffered from anxiety or depression during pregnancy have an increased risk of adverse perinatal health outcomes, and behavioural, emotional, cognitive, and motor problems in early childhood [20,21]. It is also shown that the infant cortisol stress response is altered if the mother suffered from depression during pregnancy [22]. One meta-analysis showed an association of depression during pregnancy with preterm birth and low birth weight [23]. Another more recent meta-analysis showed that depression during pregnancy is associated with premature delivery, but did not find associations with birth weight, neonatal intensive care unit admissions, preeclampsia, gestational age or Apgar scores [24].

Overall, in clinical practice and literature, pregnant women express a strong preference for non-pharmacologic treatment of depression over antidepressant medication [25]. Hence, cognitive behavioural therapy (CBT) could be a good alternative for SSRI use during pregnancy. 
According to a recent meta-analysis there is strong evidence that CBT interventions are effective for preventing depressive relapse during the perinatal period [26]. A recent follow-up study showed that preventive cognitive therapy (PCT) has long-term effects in preventing depressive relapse in patients with recurrent depression for over 5.5-10 years after the sessions ended [27, 28]. This preventive psychological strategy therefore seems promising in preventing depressive relapse, presumably also during pregnancy. Moreover, a recent study in the UK among non-pregnant patients showed that tapering antidepressants with therapy was as effective as continuation of antidepressants (Hazard Ratio 0.89) [29]. Nevertheless, further investigation is necessary to assess effectiveness of tapering antidepressants with added PCT during the perinatal period.

In conclusion, pregnant women and their clinicians face a dilemma, which is widely experienced in current practice [30]. At present, there are no suitable data available to guide evidence based decisions on SSRI continuation or discontinuation during pregnancy [31]. Both the National Institute for Health and Clinical Excellence in the United Kingdom (NICE) guideline [32], and American Psychiatric Association (APA) [33] therefore recommend to discuss both possibilities with women. The recently developed Dutch multidisciplinary guideline advises to continue SSRI use during pregnancy, and furthermore advises a hospital delivery and neonatal observation based on the increased risk and the severity of the (rare) condition of PPHN and prevalence (25-30\%) of children with neonatal abstinence after maternal SSRI use [34]. Nonetheless, the need of randomized trials was stressed. Indeed, existing studies are observational and therefore their results do not fully allow causal inference nor definite conclusions for practice.

\section{Trial objectives}

In this randomized controlled trial (RCT), the effect of preventive cognitive therapy (PCT) with guided tapering of SSRIs in early pregnancy will be compared to continuation of SSRIs during pregnancy. We will study effects on both mother and child with a pragmatic approach. The expectation is that tapering of SSRIs with added PCT does not increase the risk of clinically relevant maternal relapse or recurrence ${ }^{1}$ of depression or onset of anxiety disorders during pregnancy up to three months postpartum in excess of [absolute] $15 \%$ compared to continuation of SSRIs. If so, discontinuation is deemed non-inferior with regard to relapse/recurrence risk. Furthermore, we expect that tapering of SSRIs is better than continuation of SSRIs with respect to child development. Finally, but not unimportantly, we hypothesize that discontinuation will decrease total costs per woman and child on a 3 months and projected long term base, assuming no relevant effects of discontinuation on the mother and no effects on the child are found.

\section{Methods/Design \\ Design \& setting}

The Stop or Go study is a pragmatic multi-centre randomized controlled non-inferiority trial (RCT) in obstetric care. Women will be recruited during their first prenatal visit in midwifery practices (first echelon care) and hospitals (second and third echelons care), or through advertisement in (social) media. After inclusion, women will be randomly allocated into two groups: STOP or GO. Both groups will receive regular assessments throughout their pregnancy and up to 3 months post-partum. Permission will be asked to contact the Centre of Childhood (CJG) at 24 months after delivery for information on the development of the child. Total duration of the study for participants will therefore be 30 months. In Fig. 1 an overview of the study design and main procedures is shown.

\section{Participants}

Women who are less than 16 weeks pregnant and use a SSRI primarily for depressive disorder, and are currently at least in remission or recovered [35], are invited to participate in the trial. Exclusion criteria are multiple pregnancy, as these women have a markedly increased obstetric risk, thereby threatening the homogeneity of the study population and thus potentially complicate the statistical analysis, and insufficient proficiency in Dutch or English, since our intervention is not yet available in other languages. Also, women will be excluded with severe medical conditions, such as oncology-related conditions or conditions that need urgent medical interventions, which involve treatment decisions overriding research participation. Exclusion criteria related to mental health are: current mania or hypomania or a history of bipolar illness, suicidality and serious self harm, any psychotic disorder (current and previous), current alcohol or drug misuse, predominant anxiety disorders and personality disorders that require psychotherapeutic treatment for more than 2 sessions a month.

\section{Assessment of eligibility}

After informed consent is obtained, a pre-assessment interview will be conducted, with the Structured Clinical Interview for DSM-disorders (SCID) [36] and the Hamilton Depression Rating Scale (HDRS) [37] to assess major DSM-IV Axis I psychiatric diagnoses and actual remission status and depressive symptoms respectively. Before randomization, study researchers will contact the medical professional who prescribed the SSRI medication to inform the professional about the study and discuss exclusion criteria as described above for study participation. 
Recruitment through midwifery practices (first echelons care) and hospitals (second and third echelons care), and approaching the general population using (social) media.

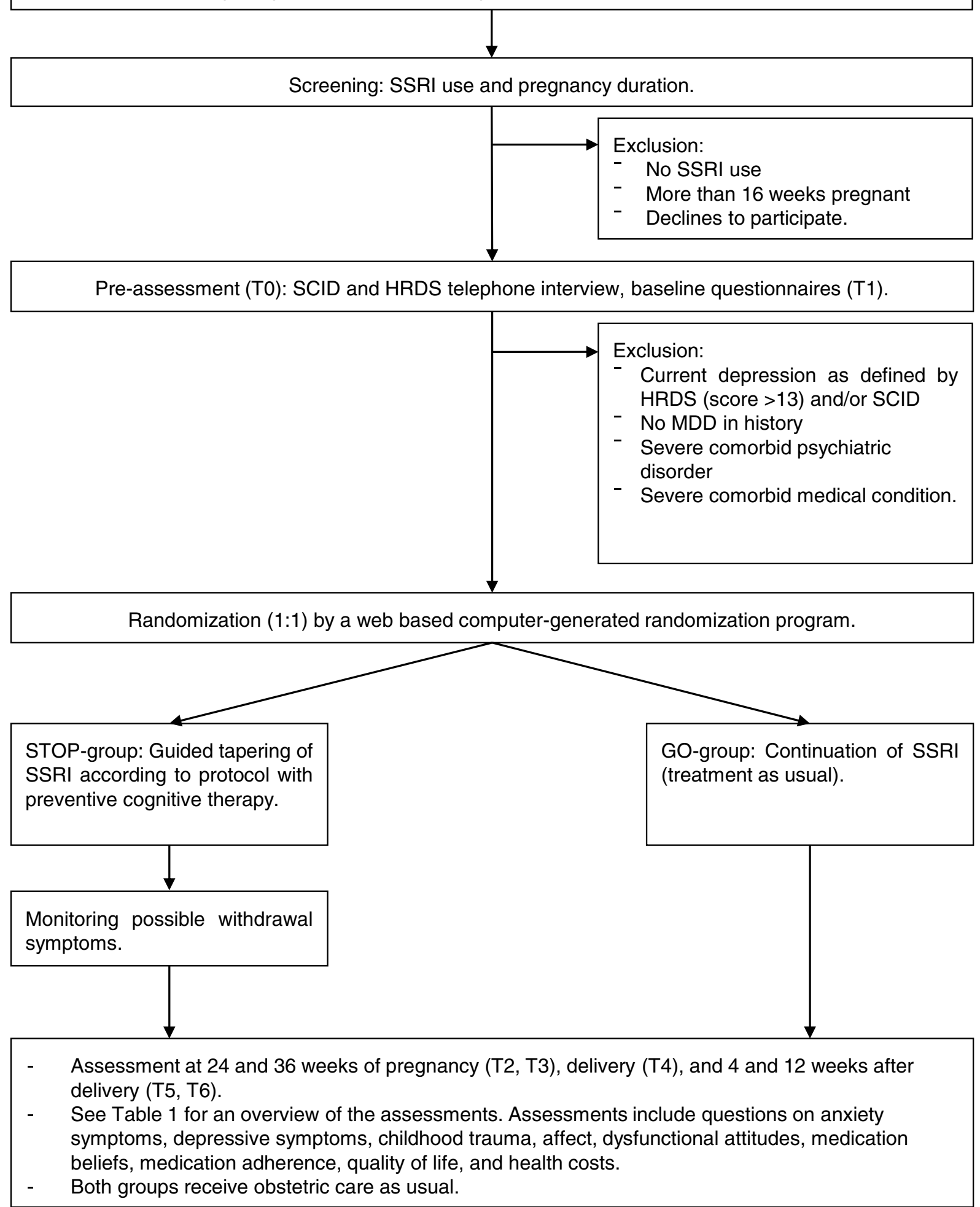

Fig. 1 Study flow chart 


\section{Randomisation}

Two hundred women will be randomized in a 1:1 allocation ratio to either the intervention arm (STOP) or the care as usual arm (GO). Randomization will be done with a web based computer-generated randomization schedule (a validated TENALEA Clinical Trial Data Management System; http://www.formsvision.com/) using permuted blocks of random size with a maximum of 16 and stratified for the number of previous depressive episodes (dichotomized). Based on a recent review [35], the participants are divided into groups of participants with 3 or less previous depressive episodes, versus 4 or more. Allocation of participants is concealed for study researchers.

\section{Interventions}

\section{Tapering SSRI}

Women assigned to discontinuation of SSRIs will be referred to a psychiatrist trained in guiding tapering of SSRIs during pregnancy. They will plan and carry out SSRI discontinuation using an expert-based discontinuation protocol [38]. The aim is to taper the use of SSRIs within four weeks, depending on patient preferences and on drug characteristics (e.g., half-life in the body). There are no restrictions on the use of medication like sleeping pills, paracetamol, and mild tranquillizers. All co-medication will be monitored during the study period.

\section{Preventive cognitive therapy}

Trained psychologists will provide preventive cognitive therapy in the discontinuation arm. This psychological intervention has proven to be effective in relapse prevention [27, 39-42]. The current manual was evaluated in previous studies [27, 38, 41, 43].

The intervention will be applied through VSee (http:// www.vsee.com), a HIPAA-compliant telehealth app. Several studies demonstrated that psychological intervention as applied by telephone support is effective and there is some evidence that it might be effective to decrease postpartum depressive symptomatology [44-47]. Although not tested during pregnancy, there are indications that antenatal telephone or online therapy is effective and convenient [48].

The preventive psychological intervention consists of a minimum of eight weekly VSee sessions. These sessions are led by professional psychologists trained in cognitive behavioural therapy and may occur at any time of the day. The focus of the sessions is on identifying and teaching the participants to challenge dysfunctional beliefs, enhance recall of positive feelings and cognitions and a personal prevention plan is developed in which it is specified how the participant can prevent a depressive episode in the future. For each session the participant will receive some assignments of approximately $10 \mathrm{~min}$ per day. Treatment adherence will be monitored.

\section{Care as usual}

Women assigned to continuation of SSRIs (GO) obtain usual care. They will be instructed to consult their doctor as they regularly do, in line with the pragmatic nature of the study. All the care that is provided will be monitored.

\section{Outcome measures \\ Mother}

Primary outcome of this trial is (cumulative) incidence of relapse or recurrence of a depressive episode (as defined by the SCID-I [36]) during pregnancy and up to 12 weeks postpartum. The SCID-I is assessed at baseline (T0) and 12 weeks postpartum (T6). If - based on assessment with the HDRS at fixed time-points - relapse/recurrence is suspected, the SCID-I will be performed intermittently.

For registration of severity of depressive symptoms, the HDRS will telephonically be assessed additionally, at baseline (T0), at 36 weeks of gestation (T3), and 12 weeks postpartum (T6), and also intermittently, if necessary [37]. When the HDRS at any stage turn out above cut-off scores, the participant will be called one week after initial measurement. The HDRS will be repeated to confirm or reject elevated scores. An adjusted telephonic version of the everyday problem checklist (EPCL) and pregnancy related life events will be assessed during each telephonic measurement (T0, T2, T3, T5 and T6).

Women will be asked to fill in questionnaires during five occasions: baseline (T1), 24 and 36 weeks of gestation (T2 and T3), and 4 and 12 weeks postpartum (T5 and T6). The questionnaires differ in composition at the five measurement moments, as shown in Table 1. During these occasions, participants are variably asked to report on anxiety symptoms (Dutch version of the State Trait Anxiety Inventory STAI), short and long version $[49,50])$, depressive symptoms (the Dutch version of the Edinburgh Postnatal Depressions Scale; EPDS [51]), childhood trauma (Childhood Trauma Questionnaire; CTQ [52]), affect (the International Short-Form of the Positive and Negative Affect Schedule; I-PANAS-SF [53]), dysfunctional attitudes (Dysfunctional Attitude Scale; DAS [54]), medication beliefs (Beliefs about Medicines Questionnaire; BMQ [55]), medication adherence (Medication Adherence Rating Scale; MARS [56]) and Quality of Life (EQ-5D-5L [57]). Socioeconomic position, ethnicity, smoking behaviour, alcohol use, family history and information on previous pregnancies and family size will be assessed using the Mind2Care questionnaire [58], a screen-and-advice instrument to detect mental health problems among pregnant women.

Health care cost data is registered using the TIC-P [59]. This instrument allows reliable recall over the past 
Table 1 Assessment per measurement moment

\begin{tabular}{|c|c|c|c|c|c|c|c|c|c|}
\hline & Method & T0 & T1 & $\mathrm{T} 2$ & T3 & T4 & T5 & T6 & $\mathrm{T7}$ \\
\hline Clinical Diagnostic Interview (SCID-I) & Int & $x$ & & $\ldots$ & $\ldots$ & $\ldots$ & $\ldots$ & $x$ & \\
\hline Depressive symptoms (HDRS) & Int & $x$ & & $\ldots$ & $x$ & $\ldots$ & $\ldots$ & $x$ & \\
\hline Peripartum depression (EPDS) & $S R$ & & $x$ & $x$ & $x$ & & $x$ & $x$ & \\
\hline Anxiety (STAl) & SR & & $x$ & $x$ & $x$ & & $x$ & $x$ & \\
\hline Affect (I-PANAS-SF) & SR & & $x$ & $x$ & $x$ & & $x$ & $x$ & \\
\hline Attitudes (DAS) & SR & & $x$ & $x$ & $x$ & & & & \\
\hline Daily hassles & Int & $x$ & & $x$ & $x$ & & $x$ & $x$ & \\
\hline Life events & Int & $x$ & & $x$ & $x$ & & $x$ & $x$ & \\
\hline $\begin{array}{l}\text { Sociodemographic \& -economic } \\
\text { factors (Mind2Care) }\end{array}$ & SR & & $x$ & & & & & & \\
\hline Substance use (Mind2Care) & $S R$ & & $x$ & $x$ & $x$ & & $x$ & $x$ & \\
\hline Medication use & Int & $x$ & & $x$ & $x$ & & $x$ & $x$ & \\
\hline Medication adherence & $S R$ & & $x$ & & & & & & \\
\hline Medication beliefs & $S R$ & & $x$ & & & & & & \\
\hline Childhood trauma (CTQ) & $S R$ & & & $x$ & & & & & \\
\hline Quality of Life (EQ-5D-5L) & SR & & $x$ & $x$ & $x$ & & $x$ & $x$ & \\
\hline Health care consumption (TIC-P) & SR & & $x$ & & $x$ & & $x$ & $x$ & \\
\hline Pregnancy related outcomes & CG & & & & & $x$ & & & \\
\hline Neural development (GM) & ME & & & & & & & $x$ & \\
\hline Child behaviour (CBCL) & SR & & & & & & & & $x$ \\
\hline Cortisol (hair strands) & BM & & $x$ & & & $x$ & & $x$ & \\
\hline Buccal swab & BM & & $x$ & & & & & $x$ & \\
\hline Blood sample & BM & & & & & & & $x$ & \\
\hline Meconium (SSRI concentration) & BM & & & & & $x$ & & & \\
\hline Breast milk (SSRI concentration) & BM & & & & & $x$ & & & \\
\hline
\end{tabular}

Int interview, SR self report, CG caregiver, BM biological materials, T0 pre-assesment, $T 1$ baseline, $T 224$ weeks of gestation, $T 336$ weeks of gestation, $T 4$ delivery,

T5 4 weeks postpartum, T6 12 weeks postpartum, T7 18 months postpartum

six months [60]. We will adapt scoring for 'normal' absenteeism and sickness leave for pregnant and recently delivered women. Care will be taken for secondary effects on child-care for other children (if present) in case of postpartum hospitalisation.

Using the Discontinuation Emergent Signs and Symptoms checklist (DESS) [61], the discontinuation group will be monitored by telephone weekly during tapering, to collect information about dosages and potential symptoms of withdrawal. Both groups will receive telephonic monitoring of medication use, including psychiatric co-medication, at 24 and 36 weeks of gestation (T2 and T3) and 4 and 12 weeks postpartum (T5 and T6).

Alongside the self-report measures, several sources of biological materials will be collected during the study. At baseline, immediately after delivery and 12 weeks postpartum (T1, T4 and T6) we will collect maternal hair strands to measure cortisol levels. Hair cortisol is a validated biomarker for long-term cortisol exposure and makes it possible to create a timeline of cortisol exposure during follow-up [62]. At baseline a maternal buccal swab will be collected in order to enable epigenetic and pharmacogenetic analysis. Maternal blood sampling will be performed 12 weeks postpartum (T6) to enable additional epigenetic and pharmacogenetic testing, but also for measurement of SSRI concentration and immunological factors.

\section{Health care professional}

We will send a Case Report Form (CRF) to the participant's obstetric caregiver, either a midwife or a gynaecologist, to request information about the pregnancy and delivery. Complications during pregnancy and delivery, such as hypertensive disorders or pregnancy, foetal growth retardation, preterm labour, induced labour and caesarean section will be registered as well as information about the neonate (e.g., Apgar scores, birth weight, congenital malformations and admission to paediatric ward). 


\section{Child}

At 12 weeks postpartum we will perform a General Movements (GM) assessment by taking video recordings at home [63]. This assessment method evaluates the function of the young nervous system.

GMs are spontaneous movements that are present from early foetal life onwards until the end of the first half-year of life. GMs are complex, occur frequently and last long enough to be observed properly. If the nervous system is impaired, GMs lose their complex and variable character and become monotonous and poor [64].

For mapping of the SSRI exposure of the newborn, samples of meconium and breast milk (if breastfeeding) will be collected. SSRI in meconium will be measured by a validated method according to LCH guidelines on LC-MS/ MS [65]. If feasible, hair strands and a buccal swab of the newborn will be collected at 12 weeks after birth (T6).

Long-term follow-up includes the well-established, reliable and valid Child Behaviour Check List 1.5-5 years, including the Caregiver Teacher Report Form (C-TRF) and the Language Development Survey (LDS) at 18 months postpartum [66]. Also, permission will be asked to obtain routine data from Centres for Childhood (CJG) until 24 months (in particular on length gain, weight gain, normal development, and any information on abnormal behavioural development).

\section{Sample size}

Sample size calculation is based on the main aim of this study, which is to demonstrate non-inferiority of preventive CT with guided discontinuation of SSRIs (STOP) compared to continuation (GO), with respect to relapse or recurrence of a depressive episode up to 3 months postnatal. We will use a non-inferiority margin (tolerance threshold, 'delta') of $15 \%$. This is based on the assumption that this excess relapse (taking into account the possibility of restoring SSRI treatment) is still in balance with the expected beneficial effects of discontinuation of SSRI for the remaining mothers. We also anticipate that this balance is acceptable for women.

With this non-inferiority margin, and the assumption that the overall absolute risk of relapse will be around $15 \%$ [67], we need 178 women, given alpha .025, power $80 \%$, and a one-sided test. To account for some attrition, we aim to include 200 women in total. Given this sample size, we have sufficient power to demonstrate small to moderate effect sizes of .42 or over on continuous secondary outcomes. With respect to dichotomous secondary outcomes, we will be able to detect odds ratios of 1.5 or over when the base probability is .5 .

\section{Statistical analysis}

Analysis will primarily be carried out according to the intention-to-treat principle, i.e., the participants will be analysed according to their randomized allocation, regardless of the actual interventions received by the participant. Supplementary, we will perform analyses per protocol, i.e., according to actual SSRI use, irrespective of randomized arm.

The primary outcome, risk (cumulative incidence up to 3 months postnatal) of relapse of depression, will be compared between the randomized groups. Differences will be assessed statistically using a one-sided Chi-Square Test at a significance level of .025 and will be presented as a risk difference. The remainder of statistical tests will be performed two-sided at a significance level of .05.

Time to relapse will be compared between the randomized groups using survival analysis. Kaplan-Meier curves will be constructed and differences will be tested using the log-rank test. A Cox proportional hazard model will be used to calculate hazard ratios

Continuous outcomes, e.g., the General Movements scores at 3 months, will be compared between the groups using the unpaired $t$-test. Categorical secondary outcomes, e.g., obstetric complications, will be tested using Chi-Square Tests. For the continuous variables and categorical variables that are assessed more than twice, we will deploy linear mixed models and generalized linear mixed models respectively. These models use all available data (do not exclude persons with missing values) under the assumption of data being missing at random, and account for within-subject correlation over time. If despite randomization prognostically important factors differ between the groups, they will be adjusted for in supplemental analyses by including these factors in the pertaining regression models.

Subgroup analyses will be undertaken according to: Dutch/non-Dutch, nulliparous/multiparous, yes/no history depressive disorder and/or anxiety disorder, yes/no comorbid anxiety symptoms or disorder. All effect parameters will be supplied with a $95 \%$ confidence interval.

\section{Economic evaluation}

In the present study we will also evaluate the outcome in the two study groups (Stop and Go) from a societal, economic perspective. It is therefore important to weigh cost savings for both groups against their clinical value. If relapse/recurrence incidence is within the predefined threshold (15\%), hence non-inferiority is confirmed; a straightforward cost minimization analysis will be executed focussing on cost savings. However, successful tapering of SSRIs will reduce SSRI use for years. Hence, with a sensitivity analysis on maternal effects and costs we will project cost estimations for 10 years. We expect that the upfront investment in PCT for women with previous psychiatric disorders will then be balanced by reduced SSRI use and less healthcare consumption. A previous RCT in a non-pregnant population demonstrated 
that a brief CT intervention is cost effective in remitted depressed individuals that stop antidepressants, compared to continuation of antidepressants [68].

If, however, relapse/recurrence incidence is higher than the predefined tolerance threshold, thus discontinuation is clinically inferior and rejected, a cost-effectiveness analysis will be executed as primary analysis, which estimates the costs avoided per additional relapse. This is the opposite of the extra costs per prevented relapse, if the starting point would have been no SSRI, and starting SSRI would be considered. Regardless the relapse outcome, we will conduct a cost utility analysis which estimates the impact of SSRI on the costs per Quality Adjusted Life Year (QALY), at least with a 3 month time horizon.

\section{Discussion}

The use of SSRIs during pregnancy remains a clinical dilemma for both clinicians and patients. Given the increase of SSRI use among pregnant women and studies reporting conflicting results $[7,8,11-14,18,19]$, there is dire need of randomized controlled trials investigating the use of SSRIs during pregnancy. This study will be the first to investigate the effect of preventive cognitive therapy with guided tapering of SSRIs from early pregnancy onwards as compared to continuation of SSRIs during pregnancy. Additionally, the study focuses on child outcomes and cost effectiveness.

Previous studies on relapse prevention showed promising results for tapering antidepressants with added relapse prevention [29]. Preventive cognitive therapy moreover showed promising long-term effects in nonpregnant women with a history of depression [27, 28]. Preventive cognitive therapy with guided tapering of antidepressants may therefore be a good alternative for SSRI use during pregnancy.

To our knowledge, no randomized controlled trials have been performed during pregnancy that investigated alternative treatment options versus SSRI use. This may be the result of the complex ethical situation of studies in pregnant women who are taking SSRIs and must be willing to either taper or continue SSRI use. Logistics of a nationwide randomized controlled trial are also difficult in a multidisciplinary setting. Although a multidisciplinary guideline exists, health care givers still have different views on best practice and therefore give different advices to their patients. This study will therefore be as pragmatic as possible, while still providing the intervention in a protocoled manner.

Results of this study will be published and will contribute to further development of (international) guidelines. The results will provide a first step in giving pregnant women an answer to the question whether it is better to stop or to continue the use of SSRIs during pregnancy.

\section{Ethics approval and consent to participate}

The Medical Ethical Commission of the Erasmus Medical Center approved this study. Participants will sign informed consent form before participation.

\section{Endnotes}

${ }^{1}$ Relapse is defined as the re-emergence of depressive symptoms during the remission phase, but before full recovery. Recurrence is defined as the onset of a new depression episode during the recovery phase or long remission phase.

\begin{abstract}
Abbreviations
BMQ: beliefs about medicines questionnaire; CBT: cognitive behavioural therapy; CJG: centres for childhood; CRF: case report form; CTQ: childhood trauma questionnaire; C-TRF: caregiver teacher report form;

DAS: dysfunctional attitude scale; DESS: discontinuation emergent signs and symptoms checklist; EPCL: everyday problem checklist; EPDS: Edinburgh postnatal depressions scale; EQ-5D-5L: quality of life questionnaire;

GM: general movements; HDRS: Hamilton depression rating scale; I-PANASSF: International short-form of the positive and negative affect schedule; LDS: language development survey; MARS: medication adherence rating scale; PCT: preventive cognitive therapy; PPHN: persistent pulmonary hypertension of the neonate; QALY: quality adjusted life year; RCT: randomized controlled trial; SCID-1: structured clinical interview for DSM-disorders axis 1; SSRI: selective serotonin reuptake inhibitors; STAI: State trait anxiety inventory.
\end{abstract}

Competing interests

The authors declare that they have no competing interests.

\section{Authors' contributions}

MPLB, CLHB, HB, GJB and WH were responsible for the development of the study and the funding. MPLB is the principal investigator, responsible for the coordination of the project. CLHB designed the intervention. CNV proposed the child outcome assessment and will be responsible for the analysis of this part of the study. JJD and HWT are responsible for the obstetric and midwifery aspect of the study. MEB and NMM are responsible for further development of study protocol details and ethical approval. Data gathering will be done by MEB and NMM. All authors read and approved the final manuscript.

\section{Funding}

This work is supported by a grant from the Netherlands Organization for Health Research and Development (836021011). Additional funding is available from the Erasmus Medical Centre, Department of Psychiatry.

\section{Author details}

'Department of Clinical Psychology, Utrecht University, Heidelberglaan 1, 3584 CS Utrecht, The Netherlands. ' 2 Department of Psychiatry, Erasmus Medical Centre, 's Gravendijkwal 230, 3015 CE Rotterdam, The Netherlands. ${ }^{3}$ Department of Obstetrics and Gynaecology, Erasmus Medical Centre, 's Gravendijkwal 230, 3015 CE Rotterdam, The Netherlands. ${ }^{4}$ Department of Pediatrics, University Medical Centre Groningen, Hanzeplein 1, 9713 GZ Groningen, The Netherlands. ${ }^{5}$ Department of obstetrics and birth care, Hogeschool Rotterdam, Rochussenstraat 198, 3015 EK Rotterdam, The Netherlands. ${ }^{6}$ Department of General Practice, University Medical Centre Groningen, Antonius Deusinglaan 1, 9713 AV Groningen, The Netherlands. ${ }^{7}$ Department of Child and Adolescent Psychiatry, Erasmus Medical Centre Sophia Childrens Hospital, Wytemaweg 80, 3015 CN Rotterdam, The Netherlands. 


\section{References}

1. Andrade SE, Raebel MA, Brown J, Lane K, Livingston J, Boudreau D, et al. Use of antidepressant medications during pregnancy: a multisite study. Am J Obstet Gynecol. 2008;198(2):194. doi:10.1016/j.ajog.2007.07.036. e1-5.

2. Charlton RA, Jordan S, Pierini A, Garne E, Neville AJ, Hansen AV, et al. Selective serotonin reuptake inhibitor prescribing before, during and after pregnancy: a population-based study in six European regions. BJOG. 2015; 122(7):1010-20. doi:10.1111/1471-0528.13143.

3. Bakker MK, Kolling P, van den Berg PB, de Walle HE, de Jong van den Berg LT. Increase in use of selective serotonin reuptake inhibitors in pregnancy during the last decade, a population-based cohort study from the Netherlands. Br J Clin Pharmacol. 2008;65(4):600-6. doi:10.1111/j.1365-2125 2007.03048.x.

4. Ververs T, Kaasenbrood H, Visser G, Schobben F, de Jong-van den Berg L, Egberts T. Prevalence and patterns of antidepressant drug use during pregnancy. Eur J Clin Pharmacol. 2006;62(10):863-70. doi:10.1007/s00228006-0177-0.

5. Quispel C, Schneider TAJ, Bonsel GJ, Lambregtse-van den Berg MP. An innovative screen-and-advice model for psychopathology and psychosocial problems among urban pregnant women: an exploratory study. J Psychosom Obstet Gynecol. 2012;33(1):7-14.

6. Gynaecologie NVvOe. Richtlijn: SSRI-gebruik in de zwangerschap en tijdens de lactatie. 2012

7. Cohen LS, Altshuler LL, Harlow BL, Nonacs R, Newport DJ, Viguera AC, et al. Relapse of major depression during pregnancy in women who maintain or discontinue antidepressant treatment. JAMA. 2006;295(5):499-507. doi:10. 1001/jama.295.5.499

8. Yonkers KA, Gotman N, Smith MV, Forray A, Belanger K, Brunetto WL, et al. Does antidepressant use attenuate the risk of a major depressive episode in pregnancy? Epidemiology. 2011;22(6):848-54. doi:10.1097/EDE. Ob013e3182306847.

9. De Vera MA, Berard A. Antidepressant use during pregnancy and the risk of pregnancy-induced hypertension. Br J Clin Pharmacol. 2012;74(2):362-9. doi:10.1111/j.1365-2125.2012.04196.x.

10. Kurki T, Hiilesmaa V, Raitasalo R, Mattila H, Ylikorkala O. Depression and anxiety in early pregnancy and risk for preeclampsia. Obstet Gynecol. 2000; 95(4):487-90.

11. Grigoriadis S, VonderPorten EH, Mamisashvili L, Roerecke M, Rehm J, Dennis $\mathrm{CL}$, et al. Antidepressant exposure during pregnancy and congenital malformations: Is there an association? A systematic review and metaanalysis of the best evidence. J Clin Psychiatry. 2013;74(4):e293-308. doi:10. 4088/JCP.12r07966

12. Furu K, Kieler H, Haglund B, Engeland A, Selmer R, Stephansson O, et al. Selective serotonin reuptake inhibitors and venlafaxine in early pregnancy and risk of birth defects: population based cohort study and sibling design. BMJ. 2015;350:h1798. doi:10.1136/bmj.h1798.

13. Kieler H, Artama M, Engeland A, Ericsson O, Furu K, Gissler M et al. Selective serotonin reuptake inhibitors during pregnancy and risk of persistent pulmonary hypertension in the newborn: Population based cohort study from the five Nordic countries. BMJ (Online). 2012;344(7842). doi:10.1136/bmj.d8012

14. Huybrechts KF, Bateman BT, Palmsten K, Desai RJ, Patorno E, Gopalakrishnan $C$, et al. Antidepressant use late in pregnancy and risk of persistent pulmonary hypertension of the newborn. JAMA. 2015;313(21):2142-51. doi:10.1001/jama.2015.5605

15. Grigoriadis S, VonderPorten EH, Mamisashvili L, Eady A, Tomlinson G, Dennis $C L$, et al. The effect of prenatal antidepressant exposure on neonatal adaptation: a systematic review and meta-analysis. J Clin Psychiatry. 2013; 74(4):e309-20. doi:10.4088/JCP.12r07967.

16. Ross LE, Grigoriadis S, Mamisashvili L, Vonderporten EH, Roerecke M, Rehm J, et al. Selected pregnancy and delivery outcomes after exposure to antidepressant medication: A systematic review and meta-analysis. JAMA Psychiatry. 2013;70(4):436-43. doi:10.1001/jamapsychiatry.2013.684.

17. Gentile S, Galbally M. Prenatal exposure to antidepressant medications and neurodevelopmental outcomes: a systematic review. J Affective Disord. 2011;128(1-2):1-9. doi:10.1016/j.jad.2010.02.125

18. Croen LA, Grether JK, Yoshida CK, Odouli R, Hendrick V. Antidepressant use during pregnancy and childhood autism spectrum disorders. Arch Gen Psychiatry. 2011:68(11):1104-12. doi:10.1001/archgenpsychiatry.2011.73.

19. Hviid A, Melbye M, Pasternak B. Use of selective serotonin reuptake inhibitors during pregnancy and risk of autism. New Engl J Med. 2013; 369(25):2406-15. doi:10.1056/NEJMoa1301449.
20. Talge NM, Neal C, Glover V, Early Stress TR, Prevention Science Network F,

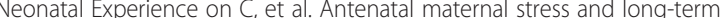
effects on child neurodevelopment: how and why? J Child Psychol Psychiatry. 2007:48(3-4):245-61. doi:10.1111/j.1469-7610.2006.01714.x.

21. Field T. Prenatal depression effects on early development: a review. Infant Behav Dev. 2011:34(1):1-14. doi:10.1016/j.infbeh.2010.09.008.

22. Oberlander TF, Weinberg J, Papsdorf M, Grunau R, Misri S, Devlin AM. Prenatal exposure to maternal depression, neonatal methylation of human glucocorticoid receptor gene (NR3C1) and infant cortisol stress responses. Epigenetics. 2008;3(2):97-106.

23. Grote NK, Bridge JA, Gavin AR, Melville JL, lyengar S, Katon WJ. A metaanalysis of depression during pregnancy and the risk of preterm birth, low birth weight, and intrauterine growth restriction. Arch Gen Psychiatry. 2010; 67(10):1012-24. doi:10.1001/archgenpsychiatry.2010.111.

24. Grigoriadis S, VonderPorten EH, Mamisashvili L, Tomlinson G, Dennis CL, Koren $\mathrm{G}$, et al. The impact of maternal depression during pregnancy on perinatal outcomes: A systematic review and meta-analysis. J Clin Psychiatry. 2013;74(4):e321-41. doi:10.4088/JCP.12r07968.

25. Battle CL, Salisbury AL, Schofield CA, Ortiz-Hernandez S. Perinatal antidepressant use: understanding women's preferences and concerns. J Psychiatr Pract. 2013;19(6):443-53. doi:10.1097/01.pra.0000438183.74359.46.

26. Sockol LE. A systematic review of the efficacy of cognitive behavioral therapy for treating and preventing perinatal depression. J Affect Disord. 2015;177:7-21. doi:10.1016/j.jad.2015.01.052

27. Bockting $\mathrm{CL}$, Schene AH, Spinhoven P, Koeter MW, Wouters LF, Huyser J, et al. Preventing relapse/recurrence in recurrent depression with cognitive therapy: a randomized controlled trial. J Consult Clin Psychol. 2005;73(4): 647-57. doi:10.1037/0022-006X73.4.647.

28. Bockting $C L$, Smid NH, Koeter MW, Spinhoven P, Beck AT, Schene AH. Enduring effects of preventive cognitive therapy in adults remitted from recurrent depression: a 10 year follow-up of a randomized controlled trial. J Affect Disord. 2015;185:188-94. doi:10.1016/j.jad.2015.06.048.

29. Kuyken W, Hayes R, Barrett B, Byng R, Dalgleish T, Kessler D, et al. Effectiveness and cost-effectiveness of mindfulness-based cognitive therapy compared with maintenance antidepressant treatment in the prevention of depressive relapse or recurrence (PREVENT): a randomised controlled trial. Lancet. 2015;386(9988):63-73. doi:10.1016/S0140-6736(14)62222-4.

30. Ververs T, van Dijk L, Yousofi S, Schobben F, Visser GH. Depression during pregnancy: views on antidepressant use and information sources of general practitioners and pharmacists. BMC Health Serv Res. 2009;9:119. doi:10.1186/ 1472-6963-9-119.

31. Hampton T. Antidepressants and pregnancy: weighing risks and benefits no easy task. JAMA. 2006;295(14):1631-3. doi:10.1001/jama.295.14.1631.

32. Excellence NIfHaC. Antenatal and postnatal mental health: clinical management and service guidance. 2014

33. Yonkers KA, Wisner KL, Stewart DE, Oberlander TF, Dell DL, Stotland N, et al. The management of depression during pregnancy: a report from the American Psychiatric Association and the American College of Obstetricians and Gynecologists. Obstet Gynecol. 2009;114(3):703-13. doi:10.1097/AOG. Ob013e3181ba0632.

34. Levinson-Castiel R, Merlob P, Linder N, Sirota L, Klinger G. Neonatal abstinence syndrome after in utero exposure to selective serotonin reuptake inhibitors in term infants. Arch Pediatr Adolesc Med. 2006;160(2): 173-6. doi:10.1001/archpedi.160.2.173.

35. Bockting $\mathrm{CL}$, Hollon SD, Jarrett RB, Kuyken W, Dobson K. A lifetime approach to major depressive disorder: the contributions of psychological interventions in preventing relapse and recurrence. Clin Psychol Rev. 2015; 41:16-26. doi:10.1016/j.cpr.2015.02.003.

36. First MB, Spitzer RL, Gibbon M, Williams JB. Structured Clinical Interview for DSM-IV-TR Axis I Disorders, Research Version, Patient Edition. Biometrics Research. New York: New York State Psychiatric Institute; 2002.

37. Hamilton M. Rating depressive patients. J Clin Psychiatry. 1980:41(12 Pt 2):21-4.

38. Bockting $\mathrm{CL}$, Elgersma HJ, van Rijsbergen $\mathrm{GD}$, de Jonge $P$, Ormel J, Buskens $E$, et al. Disrupting the rhythm of depression: design and protocol of a randomized controlled trial on preventing relapse using brief cognitive therapy with or without antidepressants. BMC Psychiatry. 2011;11:8. doi:10. 1186/1471-244X-11-8.

39. Guidi J, Fava GA, Fava M, Papakostas Gl. Efficacy of the sequential integration of psychotherapy and pharmacotherapy in major depressive disorder: a preliminary meta-analysis. Psychol Med. 2011:41(2):321-31. doi:10.1017/S0033291710000826 
40. Vittengl JR, Clark LA, Dunn TW, Jarrett RB. Reducing relapse and recurrence in unipolar depression: a comparative meta-analysis of cognitive-behavioral therapy's effects. J Consult Clin Psychol. 2007;75(3):475-88. doi:10.1037/ 0022-006X.75.3.475.

41. Bockting CL, Spinhoven P, Wouters LF, Koeter MW, Schene AH, Group DS. Long-term effects of preventive cognitive therapy in recurrent depression: a 5.5-year follow-up study. J Clin Psychiatry. 2009;70(12):1621-8. doi:10.4088/JCP.08m04784blu.

42. Guidi J, Tomba E, Fava GA. The sequential integration of pharmacotherapy and psychotherapy in the treatment of major depressive disorder: a metaanalysis of the sequential model and a critical review of the literature. Am J Psychiatry. 2015:appiajp201515040476. doi:10.1176/appi.ajp.2015.15040476.

43. Bockting $C L$, Kok GD, van der Kamp L, Smit F, van Valen E, Schoevers R, et al. Disrupting the rhythm of depression using mobile cognitive therapy for recurrent depression: randomized controlled trial design and protocol. BMC Psychiatry. 2011;11:12. doi:10.1186/1471-244X-11-12.

44. Bee PE, Bower P, Lovell K, Gilbody S, Richards D, Gask L, et al. Psychotherapy mediated by remote communication technologies: a meta-analytic review. BMC Psychiatry. 2008:8:60. doi:10.1186/1471-244X-8-60.

45. Dennis $C L$, Kingston D. A systematic review of telephone support for women during pregnancy and the early postpartum period. J Obstet Gynecol Neonatal Nurs. 2008;37(3):301-14. doi:10.1111/j.1552-6909.2008. 00235.x.

46. Lavender T, Richens Y, Milan SJ, Smyth RM, Dowswell T. Telephone support for women during pregnancy and the first six weeks postpartum. Cochrane Database Syst Rev. 2013;7:CD009338.

47. Kingston D, Austin MP, Hegadoren K, McDonald S, Lasiuk G, McDonald S, et al. Study protocol for a randomized, controlled, superiority trial comparing the clinical and cost- effectiveness of integrated online mental health assessment-referral-care in pregnancy to usual prenatal care on prenatal and postnatal mental health and infant health and development: the Integrated Maternal Psychosocial Assessment to Care Trial (IMPACT). Trials. 2014;15:72. doi:10.1186/1745-6215-15-72.

48. Committee GEA. Australian clinical practice guidelines for depression and related disorders - anxiety, bipolar disorder and puerperal psychosis - in the perinatal period. A guideline for primary health care professionals. Melbourne: Beyondblue; 2011.

49. Spielberger CD. State-Trait Anxiety Inventory: Bibliography. 2nd ed. Palo Alto: Consulting Psychologists Press; 1989.

50. Marteau TM, Bekker $\mathrm{H}$. The development of a six-item short-form of the state scale of the Spielberger State-Trait Anxiety Inventory (STAI). Br J Clin Psychol. 1992;31(Pt 3):301-6.

51. Bergink V, Kooistra L, Lambregtse-van denBerg MP, Wijnen $H$, Bunevicius $R$, van Baar $A$, et al. Validation of the Edinburgh depression scale during pregnancy. J Psychosom Res. 2011;70(4):385-9. doi:10.1016/j.jpsychores.2010.07.008

52. Bernstein DP, Stein JA, Newcomb MD, Walker E, Pogge D, Ahluvalia T, et al. Development and validation of a brief screening version of the childhood trauma questionnaire. Child Abuse Negl. 2003;27(2):169-90.

53. Thompson R. Development and validation of an internationally reliable short-form of the Positive and Negative Affect Schedule (PANAS). J Cross Cult Psychol. 2007;38(2):227-42. doi:10.1177/0022022106297301.

54. Weissman A. Dysfunctional Attitude Scale: A validation study. Unpublished doctoral dissertation. 1979. Available at: http://repository.upenn.edu/cgi/ viewcontent.cgi?article $=2994 \&$ context=edissertations

55. Horne R. The beliefs about medicines questionnaire: the development and evaluation of a new method for assessing the cognitive representation of medication. Psychol Health. 1997;14(1):1-24. doi:10.1080/08870449908407311.

56. Horne R, Weinman J. Patients' beliefs about prescribed medicines and their role in adherence to treatment in chronic physical illness. J Psychosom Res. 1999;47(6):555-67.

57. Herdman M, Gudex C, Lloyd A, Janssen M, Kind P, Parkin D, et al. Development and preliminary testing of the new five-level version of EQ-5D (EQ-5D-5L). Qual Life Res. 2011;20(10):1727-36. doi:10.1007/s11136-011-9903-x

58. Quispel C, Bangma M, Kazemier BM, Steegers EAP, Hoogendijk WJG, Papatsonis DNM, et al. The role of depressive symptoms in the pathway of demographic and psychosocial risks to preterm birth and small for gestational age. Midwifery. 2014;30(8):919-25.

59. Bouwmans C, De Jong K, Timman R, Zijlstra-Vlasveld M, Van der FeltzCornelis C, Tan Swan S, et al. Feasibility, reliability and validity of a questionnaire on healthcare consumption and productivity loss in patients with a psychiatric disorder (TiC-P). BMC Health Serv Res. 2013;13:217. doi:10.1186/1472-6963-13-217.

60. van den Brink M, van den Hout WB, Stiggelbout AM, Putter $H$, van de Velde CJ, Kievit J. Self-reports of health-care utilization: diary or questionnaire? Int J Technol Assess Health Care. 2005;21(3):298-304.

61. Rosenbaum JF, Fava M, Hoog SL, Ascroft RC, Krebs WB. Selective serotonin reuptake inhibitor discontinuation syndrome: a randomized clinical trial. Biol Psychiatry. 1998;44(2):77-87.

62. Staufenbiel SM, Penninx BW, Spijker AT, Elzinga BM, van Rossum EF. Hair cortisol, stress exposure, and mental health in humans: a systematic review. Psychoneuroendocrinology. 2013;38(8):1220-35. doi:10.1016/j.psyneuen. 2012.11.015

63. Einspieler C, Prechtl HF. Prechtl's assessment of general movements: a diagnostic tool for the functional assessment of the young nervous system. Ment Retard Dev Disabil Res Rev. 2005;11(1):61-7. doi:10.1002/mrdd.20051.

64. Zuk L. Fetal and infant spontaneous general movements as predictors of developmental disabilities. Dev Disabil Res Rev. 2011;17(2):93-101. doi:10. 1002/ddrr.1104

65. ICH guideline. http://www.ema.europa.eu/docs/en_GB/document_library/ Scientific_guideline/2009/09/WC500002662.pdf. Accessed on 8 Feb 2016.

66. Rescorla LA. Assessment of young children using the Achenbach System of Empirically Based Assessment (ASEBA). Ment Retard Dev Disabil Res Rev. 2005;11(3):226-37. doi:10.1002/mrdd.20071.

67. Yonkers KA, Gotman N, Smith MV, Forray A, Belanger K, Brunetto WL, et al. Does antidepressant use attenuate the risk of a major depressive episode in pregnancy? Epidemiology. 2011;22(6):848-54.

68. Kuyken W, Byford S, Taylor RS, Watkins E, Holden E, White K, et al. Mindfulness-based cognitive therapy to prevent relapse in recurrent depression. J Consult Clin Psychol. 2008;76(6):966-78. doi:10.1037/a0013786.

\section{Submit your next manuscript to BioMed Central and we will help you at every step:}

- We accept pre-submission inquiries

- Our selector tool helps you to find the most relevant journal

- We provide round the clock customer support

- Convenient online submission

- Thorough peer review

- Inclusion in PubMed and all major indexing services

- Maximum visibility for your research

Submit your manuscript at www.biomedcentral.com/submit
Ciomed Central 